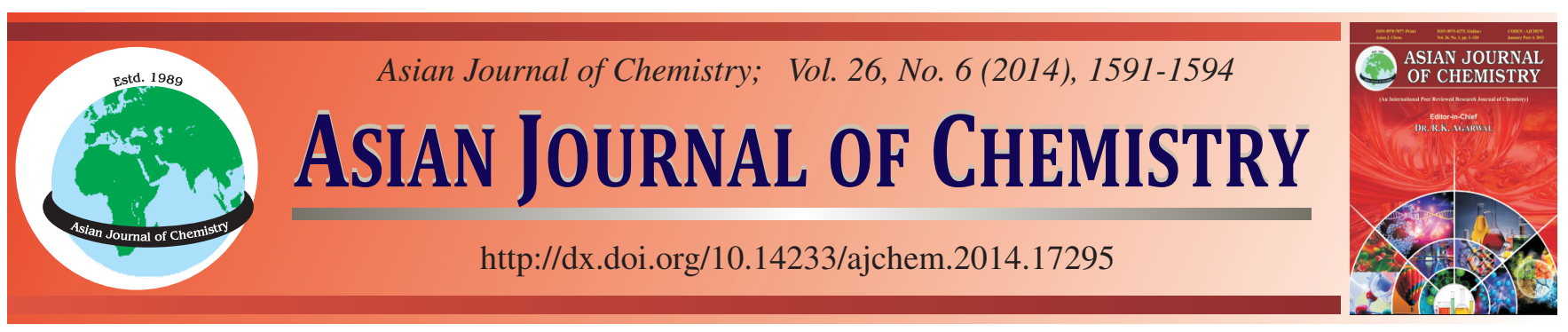

\title{
Laser Inspection for Distinguishing Real Crack from Suspected Defects of Glass Boards $\dagger$
}

\author{
Ju Yong $\mathrm{CHO}^{1}$, J $\mathrm{AE}$ Won $\mathrm{CHOI}^{2}$ and Won KweOn JANG ${ }^{1, *}$
}

${ }^{1}$ Division of Electronic, Computer and Communication Engineering, Hanseo University, Seosan-si, Chungnam-do, 356-706, Republic of Korea ${ }^{2}$ Westminster School, Simsbury, CT, 06070-1880, USA

*Corresponding author: E-mail: jwk@ hanseo.ac.kr

\begin{abstract}
We proposed an additional optical algorism to improve the functionality of the conventional in situ glass inspection system of finding out real cracks in the glass boards of LCD and OLED. The modulated pulse laser output was used as an optical probe to investigate glass plates. Two parameters of the attenuated laser pulse counting and the decrease of transmitted optical power were used to modify crack detection algorism. Compared to that of the conventional in situ glass inspection method, the detecting accuracy of real cracks was increased with the additional laser inspection method. The technical parameters for analyzing glass defects were discussed such as pulse repetition rate of the incident laser, moving speed of glass board, and attenuation of the transmitted laser power. We also discussed the field adaptation factors of focal length, spot size and wavelength of the incident laser as well as the experimental field data of the laser inspection added system.
\end{abstract}

Keywords: Fake crack, Real crack, Laser inspection, Glass board.

\section{INTRODUCTION}

The display industry has rapidly developed during last decade with the technical convolution of touch pad, smart TV and mobile phone. The expandability and applicability of display technology has changed our lifestyle from home appliance to versatile devices such as mobile touch pad, smart TV and phone. In the display product line, the glass inspection equipment has played a role of preconditioning process to supply the untainted glass board for LCD and OLED panels. According to tendency of technical diversification and improvement, the manufacturing technology of large flat panel tends to move to use higher quality glass board. In flat panel manufacturing equipments, the micro-crack inspection system of the large glass board conveyer is an important module to reduce further processing of defective product and overall cost. The optical inspection module especially provides the preliminary process before the main product processes. Many optical methods have been tried to inspect micro-cracks in silicon wafer ${ }^{1-5}$, optical substrate $^{6,7}$, metal surface ${ }^{8}$ and others where difficult to use direct inspection ${ }^{9-11}$. The other conventional inspection method of vision system with CCTV could have not offered an expected test error rate due to its insufficient accuracy. The vision system method based on the pixel variation from rapidly conveying glass board and the fake crack and the stain also generate the same pixel variation like that of real crack. This vision inspection module using CCTV executes the distinguishing function for real micro cracks from glass board defects with an algorism developed from accumulated data ${ }^{12}$. The detection algorism of vision inspection system, of course, have improved the accuracy to the considerable level, but the product field still suffer from the unexpected fault detection of fake crack and stain. Though many inspection methods have been tried to reduce the false decision and speed up the inspection, it is not easy to raise the accuracy to the level of the perfect performance under real operational environment. Considering the loss of productivity that caused by a mistake of distinguishing fake and real cracks in the glass inspection process, the additional and improved detection system is essential and burning problem.

The additional algorism and optical inspection module is proposed to reduce the loss of productivity in the product line of LCD and OLED glass boards. The optical inspection system composed of a modulated near infrared laser as a light source and an optical detection module to improve the distinguishing ability of fake and real cracks. 
In this study, we propose the newly developed laser inspection module for distinguishing fake and real cracks from suspected defects of LCD and OLED glass boards. This preliminary inspection process was performed during the glass boards moving on the conveyer system. The experimental result and theoretical explanation was analyzed considering the real operational environment.

Optical inspection based on the modulated infrared laser: The conventional vision inspection with CCTV detects the micro crack by indirect measurement. This method collects the changed pixel number and image pattern that caused by any suspicious defects of glass board moving rapidly. The shaped pattern of changed pixels needs the secondary review algorism if it should coincide with the accumulated crack shape data. However, the serious confusion may come from a fake crack that similarly shaped with real crack. The mistake could be happened due to the insufficient information about the suspicious cracks. The additional and decisional information for distinguishing real and fake cracks should be provided to prevent misjudgement.

Fig. 1 shows the transmission spectra of glass board and one of fake cracks. The largest difference between the transmissions of glass board and fake crack was around $976 \mathrm{~nm}$.

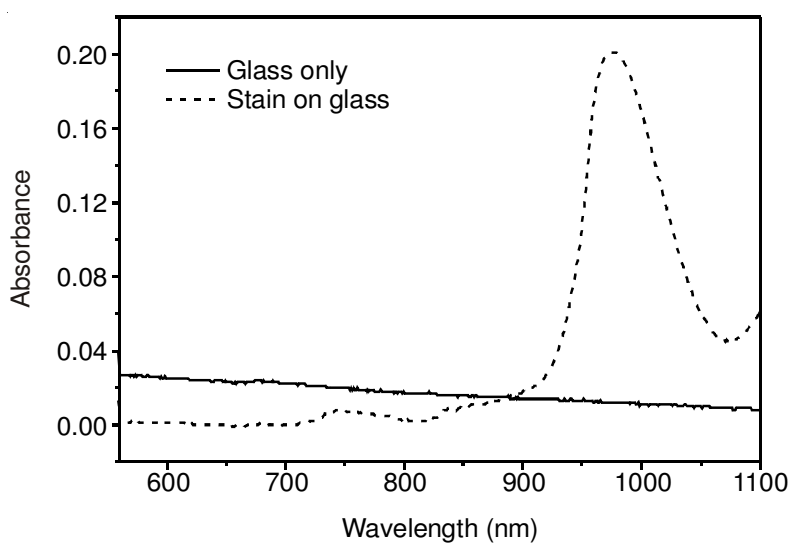

Fig. 1. Spectral absorbance of the glass board and the stain

There are many kinds of fake cracks with different transmissions. The fake crack we investigated was a transparent stain in visible region. It shows the maximum absorbance of 0.2 around $976 \mathrm{~nm}$ while the glass board was transparent at the same wavelength region. In other wavelength region, the difference of absorbance between the glass board and the stain was not over 0.035 . Many types of fake cracks show similar pattern of transmission spectra as shown in Fig. 1 even if those transmission were different at given wavelength region. It depends on the detergent for washing glass surfaces. Most corporations use different detergent and unwillingly open to the public.

Fig. 2 is the schematic illustration for real and fake crack detection. A pulse modulated near infrared laser was used for an optical signal source. In case of fake crack, the detected optical signal depends only on the transmittance of stain as shown in Fig. 1. However, in the case of real crack, the intensity of the detected optical signal can be anomalistically changed. The shape of the real crack has no pattern, but the width usually is limited to be narrower than fake crack. The transmitted laser

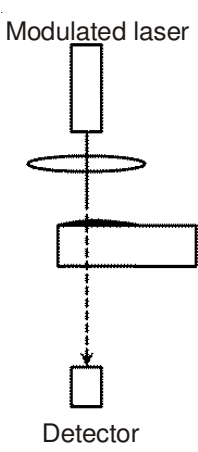

(a)

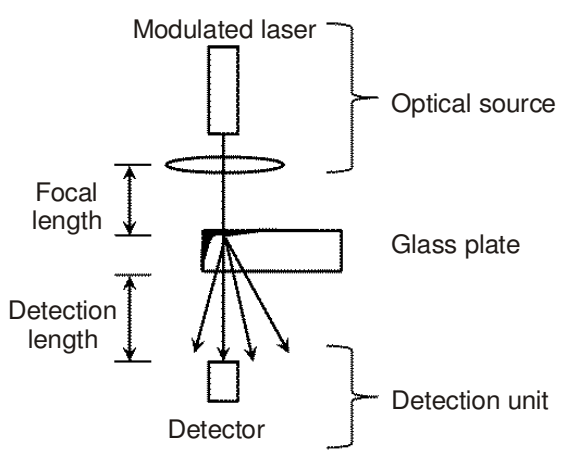

(b)
Fig. 2. Schematic diagram of (a) fake and (b) real cracks detection

pulse intensity could not be certain valued. In real application of the laser inspection method, the focal length and the detection length are floating factors depending on the installation environment of the optical inspection module. The laser inspection module must include the solid frame to maintain the operational conditions.

The modulation frequency of the pulsed laser was controlled to match up to the moving speed of glass board and the resolution of the detection device. When the moving speed of glass board was $300 \mathrm{~mm} / \mathrm{s}$, the modulation frequency of the laser was controlled to $30 \mathrm{kHz}$ to get a resolution of $0.01 \mathrm{~mm}$, in other words, the glass board moves $0.01 \mathrm{~mm}$ at that time interval. The faster moving speed or the higher resolution, the higher modulation frequency was required. In case of the vision inspection system, the higher moving speed and the higher resolution were limited by the many stepped and complicated algorism. On the other hand, the laser inspection method that proposed in this study hardly has a limitation in the choice of moving speed and resolution.

Fig. 3 is the transmitted optical signal ratio for the incident laser power with the laser wavelength. Seven wavelengths of $543,589,633,808,850,980,1064 \mathrm{~nm}$ of the incident laser were tried for the same fake crack. As shown in Fig. 3, the transmitted signal varied with the incident laser wavelength. This experimental data is useful to choose an optimal laser wavelength to distinguish the fake and real cracks. The larger the difference of transmitted optical signal ratio between fake and real cracks, the easier distinguishing is possible.

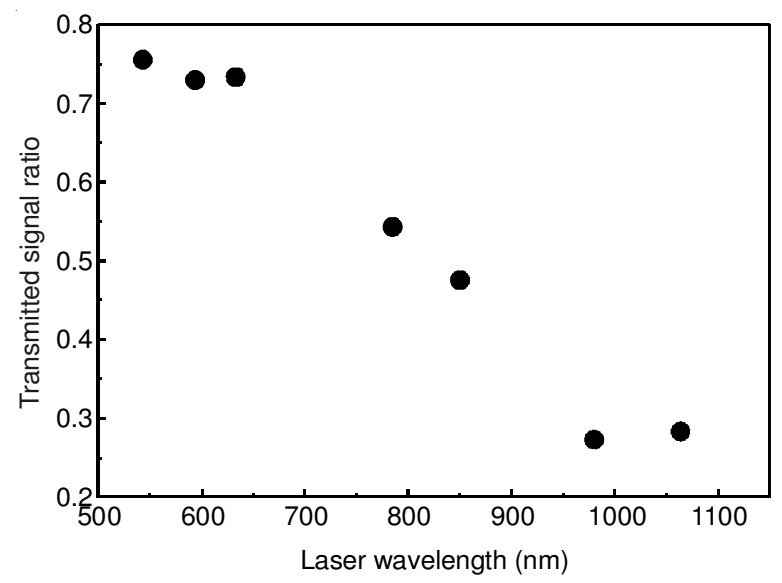

Fig. 3. Transmitted signal ratio of the stain with incident laser wavelength 
Fig. 4 shows the transmitted optical signal ratio of four different laser wavelengths of 785, 850, 980 and $1064 \mathrm{~nm}$ for the fake crack detection. The number and the shape of the attenuated data points were nearly same regardless of incident laser wavelength. The only transmitted optical signal ratio showed the wavelength dependence, which were the concerted results to Figs. 1 and 3.

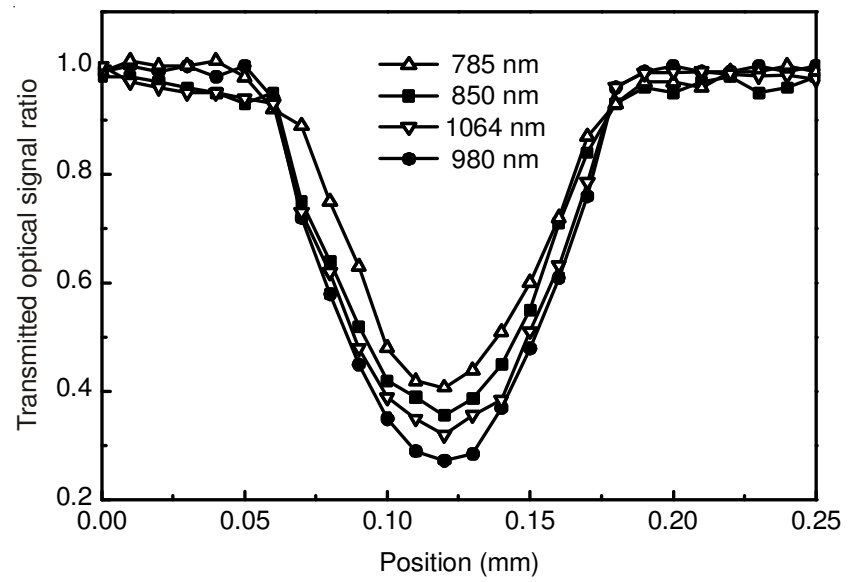

Fig. 4. Transmitted optical signal ratio of four different laser wavelengths for the fake crack detection

Fig. 5 representatively shows the theoretical explanation how to get the information of a crack width and find out the distinction between real and fake cracks. Usually the crack direction is perpendicular the moving direction of glass board as shown in Fig. 5(a). The cracked positions usually are at the edge of the glass board. Of course, there is an exception in the crack direction and position, but Fig. 5 shows the general case of real crack for easy understanding. The detected power level of laser pulses provides the distinction between true and fake cracks and the number of attenuated laser pulses indicates the width of the crack formed in the glass board. The smaller laser spot size, the clearer data points obtained.

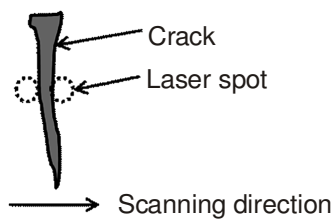

(a) Scanning crack

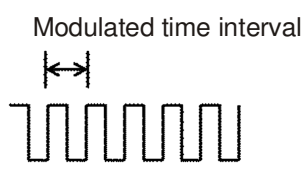

(c) Detected laser pulses

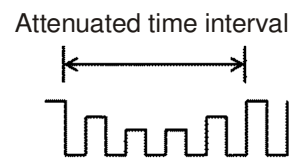

(b) Incident laser pulses

Fig. 5. (a) Scanning crack, (b) incident laser pulses and (c) detected laser pulses when the modulation frequency was applied to get the information of a crack width from the attenuated time interval

Fig. 6 is the magnified picture of a real crack formed in the edge of LCD glass board. For size estimation the glass board placed on the millimeter graticule. Case A is narrow region and case $\mathrm{B}$ is wide region of the same crack. As shown in Fig. 6, the detected attenuated time interval depends on the

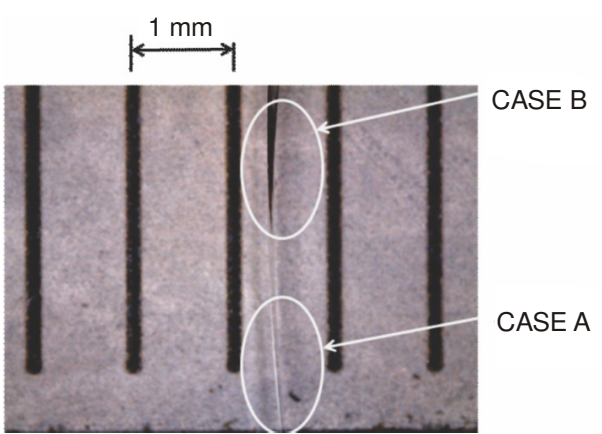

Fig. 6. Picture of a real crack formed in the LCD glass board

scanning position even the same crack. Actually, the figure of most detected crack is like case B and rarely sometimes like case $\mathrm{A}$. The modulation frequency should be raised to catch the crack of case A under high moving speed of glass board. The developed laser inspection module can easily reach to the resolution of micron by controlling modulation frequency of the laser.

Fig. 7 is the detected laser pulse signal for a real crack when the spot diameter of focused laser pulse was $0.1 \mathrm{~mm}$. The case $\mathrm{A}$ is narrow and the case $\mathrm{B}$ is wide comparing two cases. The detected optical signal data points showed the unshaped and irregular pattern. The incident laser wavelength was $1064 \mathrm{~nm}$, near infrared to get an obvious variation in transmitted optical signal ratio. The attenuated time interval of case A and case B were 0.10 and $0.15 \mathrm{~mm}$, respectively and the number of attenuated light signal were about ten and fifteen, respectively. The crack width, w, can be calculated with the equation of $\mathrm{w}=\mathrm{n} \times(\mathrm{v} / \mathrm{n})$, where $\mathrm{n}$ is the number of attenuated light signal, $\mathrm{f}$ is the modulation frequency of laser and $\mathrm{v}$ is the moving speed of glass board.

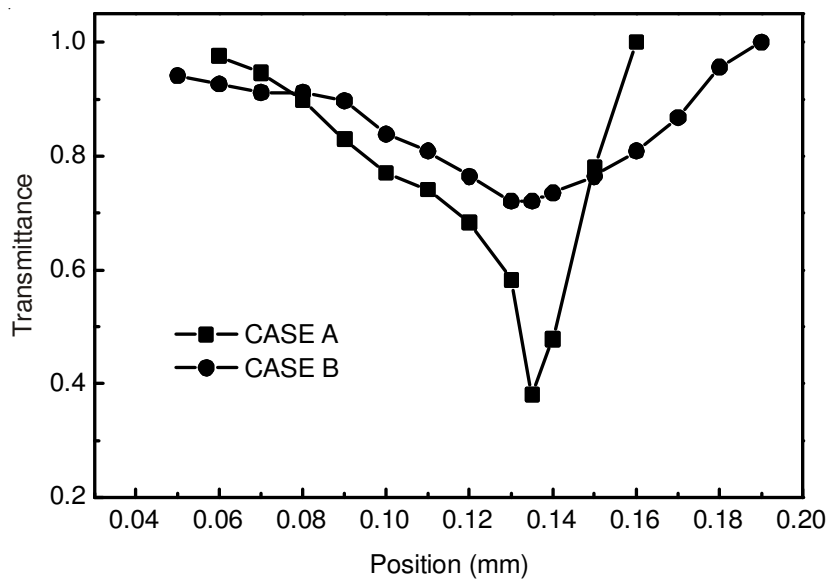

Fig. 7. Detected laser pulse signal for a real crack

\section{Conclusion}

The newly developed laser inspection method to distinguish fake and real cracks from suspicious defect in the glass boards of LCD and OLED was proposed with the experimental and theoretical results. The modulation frequency and the moving speed of the glass board was discussed in the range of around $30 \mathrm{kHz}$ and $300 \mathrm{~mm} / \mathrm{s}$. The detected optical signal transmittance for the real and fake cracks was obtained in field application of the glass board conveyer. The laser inspection 
module can be a subsidiary module to improve the detection accuracy for any suspicious defects in the glass boards. The optical inspection resolution depending on the modulation frequency was possibly up to sub-micron level at meters per second moving speed of glass board conveyer.

\section{ACKNOWLEDGEMENTS}

This research was funded by 2013 research grant of Hanseo University.

\section{REFERENCES}

1. S.-S. Ko, C.-S. Liu and Y.-C. Lin, Optik, 124, 4030 (2013)

2. C. Hilmersson, D.P. Hess, W. Dallas and S. Ostapenko, Appl. Acoust., 69, 755 (2008)
3. J.C.-H. Pan and D.H.E. Tai, Comput. Ind. Eng., 60, 16 (2011).

4. J.Y. Cho, B. Woo and W.K. Jang, Appl. Mechan. Mater., 394, 232 (2013).

5. D.-M. Tsai, C.-C. Chang and S.-M. Chao, Image Vis. Comput., 28, 491 (2010).

6. J. Shen, S. Liu, K. Yi, H. He, J. Shao and Z. Fan, Optik, 116, 288 (2005).

7. W.K. Jang, J. Photocatal. Sci., 3, 107 (2012).

8. M.M. Tehranchi, S.M. Hamidi, H. Eftekhari, M. Karbaschi and M. Ranjbaran, Sens. Actuators A, 172, 365 (2011).

9. M.S. Safizadeh and T. Azizzadeh, NDT Int., 52, 144 (2012).

10. S.-H. Kim, J.-H. Kim and S.-W. Kang, Displays, 32, 325 (2011).

11. C. Quan, S.H. Wang, C.J. Tay, I. Reading and Z.P. Fang, Opt. Commun., 225, 223 (2003).

12. EGiS-crack, Information on http://www.semisysco.com. 